\title{
Reprodutibilidade e validade do questionário Baecke para avaliação da atividade física habitual em adolescentes
}

\author{
Dartagnan P. Guedes \\ Cynthia C. Lopes \\ Joana E. R. P. Guedes \\ Luiz C. Stanganelli
}

https://doi.org/10.5628/rpcd.06.03.265

\author{
Centro de Educação Física e D esporto \\ Universidade Estadual de Londrina \\ Paraná \\ Brasil
}

\section{RESUMO}

O objetivo do estudo foi analisar indicadores associados à reprodutibilidade e à validade do Questionário de Atividade Física Habitual preconizado por Baecke (BQHPA) em amostra específica de adolescentes. A amostra foi constituída por 161 sujeitos (92 moças e 69 rapazes) com idades entre 12 e 18 anos. Reprodutibilidade foi obtida por intermédio de réplicas de aplicação do questionário, com intervalo de duas semanas, envolvendo o coeficiente de correlação intraclasse $(R)$ e a técnica de Bland e Altman (B-A). A validade foi analisada mediante comparações entre as informações apresentadas pelos adolescentes no BQHPA e por instrumento retrospectivo de autorecordação das atividades diárias (R-24h). Para tanto, utilizouse da proporção de concordância Kappa entre categorizações de acordo com a distribuição de quartis dos indicadores de atividade física. Os achados revelaram $\mathrm{R}$ entre 0,55 e 0,85 nas moças e entre 0,69 e 0,82 nos rapazes. Embora esses valores sejam semelhantes aos encontrados em outros estudos, mediante a técnica de B-A verifica-se limitada capacidade de concordância entre réplicas de aplicação do questionário. Quanto à validade, os escores de atividade física apresentados pelo BQHPA demonstraram proporção de concordância entre $33,5 \%$ e $76,6 \%$ com as informações provenientes do R-24h. Os indicadores de validação observados foram mais elevados entre os rapazes com mais idade. Em conclusão, os resultados apresentam evidências de que, em adolescentes de ambos os sexos com mais de 14 anos de idade, o BQHPA demonstra aceitáveis propriedades de medida para monitorar níveis habituais de atividade física. Em adolescentes mais jovens ( $\leq 14$ anos), os achados sugerem que o BQHPA deverá ser utilizado com algumas precauções.

\section{ABSTRACT \\ Reproducibility and validity of the Baecke questionnaire for assessing of the habitual physical activity in adolescents}

The present study was designed to analyze the reproducibility and validity of Baecke $\mathrm{Q}$ uestionnaire of $\mathrm{H}$ abitual Physical Activity (BQHPA) in specific sample of adolescents. A total of 161 subjects (92 girls and 69 boys) aged 12-17 years old were included in the study. For the assessment of reproducibility intraclass correlation coefficients and Bland-A Itman plots were computed between administrations of the questionnaire at baseline, and after two weeks. Validity was determined by comparing questionnaire against a 24 -hour recall instrument of the daily activities, which provided an estimate of the diary energy expenditure $(\mathrm{kcal} / \mathrm{kg} /$ day). Proportion of agreement ( $\mathrm{kappa}$ values) was calculated, based on categorization of the distributions of the physical activity variables into quartiles. The results showed correlation coefficients ranged from 0,55 to 0,85 in girls and 0,69 to 0,82 in boys. A lthough these data are comparable to most other self-report reproducibility studies, Bland-A Itman plots demonstrated limited capacity of agreement between test-retest of the questionnaire. In terms of validity, the scores of physical activity reported in the BQH PA showed percentage of agreement between $33,5 \%$ and $76,6 \%$ with the 24 -hour recall data. The indicators of validation observed were greater in boys older. In conclusion, these data provide evidence that, in adolescents of both sex with more than 14 years old, the BQH PA has acceptable measurement properties for monitoring levels of habitual physical activity. In youngest adolescents ( $\leq 14$ years old), our findings indicate that the BQHPA should be used with some precautions.

Key-words: measurement, evaluation, methods, accuracy, reliability, validation.

Palavras-chave: mensuração, avaliação, métodos, precisão, exatidão. 


\section{INTRODUÇÃO}

Os benefícios para a saúde associados à adoção de um estilo de vida fisicamente ativo são amplamente citados e comprovados na literatura, sobretudo no que se refere à prevenção quanto ao aparecimento e ao desenvolvimento de disfunções crônico-degenerativas (8, 31). Apesar de o maior número de estudos envolver sujeitos adultos, não existe dificuldade em selecionar evidências quanto às vantagens de os adolescentes tornarem-se adequadamente ativos fisicamente. Além dos benefícios no campo biológico (12, 30) e psicoemocionais (10, 20), importantes estudos têm procurado destacar que hábitos de prática da atividade física incorporados na adolescência podem transferir-se para as idades adultas (15, 28), o que destaca a importância de acompanhar mais proximamente os hábitos de prática da atividade física dos adolescentes.

Entendida como qualquer movimento corporal, produzido voluntariamente pelos músculos esqueléticos, que resulta em dispêndio energético acima dos níveis considerados de repouso (11), a evolução dos estudos no campo da atividade física relacionada à saúde tem motivado pesqui sadores da área a sugerirem recomendações quanto à indicação de dispêndios energéticos/ dia, independente da intensidade e do tipo de trabal ho muscular realizado, que poderiam eventualmente induzir à aquisição e à conservação de um estado de saúde positivo $(5,21,26)$. $\mathrm{Na}$ tentativa de monitorar o nível de prática habitual da atividade física em diferentes populações, encontra-se disponível uma grande variedade de métodos a serem empregados na coleta das informações (13,

32). Cada um dos métodos apresenta características próprias e, portanto, demonstram vantagens e limitações que necessitam ser consideradas quando de sua utilização. A despeito de suas limitações, entre os métodos mais freqüentemente utilizados, destacam-se os questionários, construídos com intuito de se levantar informações específicas equivalentes as atividades físicas realizadas no cotidiano (14). No entanto, as informações relacionadas à prática de atividade física reunidas mediante a aplicação de questionários podem diferir por conta da natureza e das especificações das questões apresentadas, o que deverá variar de acordo com o sexo, a idade, o desenvolvimento cognitivo e o contexto sociocultural em que os sujeitos estão inseridos, e o procedimento de tratamento dos dados direcionados ao cálculo do dispêndio energético ou do índice de atividade física $(13,14)$. Portanto, previamente à aplicação de um questionário torna-se importante analisar indicadores quanto às suas propriedades psicométricas encontradas especificamente na população em que deverá ser empregado.

Neste particular, dentre as inúmeras opções de questionários apresentadas na literatura, em função das evidências quanto ao atendimento dos critérios de praticidade, o Questionário de Atividade Física Habitual preconizado por Baecke et al. (4) (Baecke Q uestionnaire of Habitual Physical A ctivity - BQHPA) vem recebendo atenção especial dos estudiosos da área. O BQHPA tem sua origem na Holanda e vem sendo disseminado em vários países, inclusive em países de língua portuguesa (16, 17, 19). Embora al guns estudos tenham procurado apresentar indicações relacionadas à reprodutibilidade e à validade desse questionário em diferentes populações $(2,18,22,23)$, até o momento, para o nosso conhecimento, não são localizados estudos com essas características envolvendo segmentos da população jovem brasileira. Em sendo assim, o objetivo do presente estudo foi analisar indicadores associados à val idade concorrente e à reprodutibilidade mediante réplicas de aplicação do BQHPA em uma amostra de adolescentes.

\section{MATERIAL E MÉTODOS}

Para a elaboração do estudo foram selecionados estudantes regularmente matriculados em três escolas da cidade de Londrina, Paraná, Brasil. Optou-se por envolver as três escolas por conta da similaridade quanto às características administrativas (escolas da rede pública de ensino fundamental e médio), da localização geográfica ( escolas próximas e localizadas na região central do município) e da representatividade do perfil socioeconômico de seus alunos no universo de escolares que freqüentam escolas públicas no município.

Os procedimentos empregados no estudo foram aprovados pelo Comitê de Ética em Pesquisa da 
Universidade Estadual de Londrina e acompanham normas da Resolução 196/ 96 do Conselho Nacional de Saúde sobre pesquisa envolvendo seres humanos. A inclusão dos sujeitos na amostra do estudo ocorreu por desejo em participar do experimento. Para tanto, o universo de escolares matriculados nas 6a, $7 \underline{a}$ e $8^{\underline{a}}$ séries do ensino fundamental e nas $1^{\underline{a}}$ e $2^{\underline{a}}$ séries do ensino médio que frequentavam as três escolas selecionadas (1628 escolares) foram contatados, informados quanto à sua natureza e convidados a participar do estudo. Dos 230 escolares que inicialmente se interessaram em participar do estudo, 69 deles não completaram todas as etapas previstas no delineamento do estudo, sendo portanto excluídos da amostra definitiva. Dessa forma, a amostra definitiva do estudo foi constituída por 161 escolares de ambos os sexos ( $10 \%$ da base populacional), com idades entre 12 e 18 anos. Para análise das informações, considerando a amplitude da faixa etária e do equivalente nível de escolaridade dos sujeitos selecionados (ensino fundamental e médio), optou-se por segmentar a amostra em quatro grupos: (a) moças $\leq 14$ anos ( $n=59$ ); (b) moças $>14$ anos ( $n$ $=33)$; (c) rapazes $\leq 14$ anos $(\mathrm{n}=38)$; e (d) rapazes $>14$ anos $(\mathrm{n}=31)$.

O BQHPA é auto-administrado, tendo como período de referência os últimos 12 meses, estruturado por 16 questões distribuídas em três seções distintas, cada uma procurando estabelecer estimativas quanto a uma dimensão específica do nível de prática habitual de atividade física. As opções de respostas são codificadas mediante uma escala Lickert de 5 pontos, com exceção da ocupação profissional e da modalidade de esporte que prática, quando for o caso. As questões de 1 a 8 constitui a primeira seção do questionário e procuram abranger as atividades físicas diárias realizadas na escola e/ ou no trabal ho. A segunda seção do questionário envolve as questões de 9 a 12 e reúnem informações quanto às atividades esportivas, aos programas de exercícios físicos e às práticas de lazer ativo. A terceira seção do questionário visa obter indicações relacionadas às atividades de ocupação do tempo livre e de locomoção. Mediante o somatório das pontuações específicas atribuídas as questões agrupadas em cada uma das partes do questionário são estabel ecidos escores equival entes às atividades físicas na escola/ trabalho (AFOcupacional), nos esportes/ exercícios físicos/ lazer ativo (AFEsporte) e na ocupação do tempo livre/ locomoção (AFLocomoção). Por este questionário podem-se estabel ecer também, estimativas quanto ao escore da atividade física habitual (AFHabitual), mediante o somatório dos escores atribuídos a cada uma das dimensões de atividade física. Aos adolescentes que, além de freqüentar a escola também trabal havam, foi solicitado considerar aquela atividade que ocupava o mai or tempo ao longo do dia, ou seja, se o trabalho Ihe ocupasse um tempo maior que a escola, deveria optar por relatar as atividades do trabal ho. Caso contrário deveria optar por relatar as atividades realizadas na escola. Originalmente o BQHPA é apresentado em língua inglesa, o que exigiu dos autores do estudo sua tradução e adaptação para o idioma português. Versões original e traduzida do questionário foram encaminhadas a dois outros estudiosos na área, fluentes em língua inglesa e portuguesa, na tentativa de apreciarem a forma de apresentação das questões no idioma português.

Para a aplicação do BQHPA os adolescentes foram reunidos em grupos de 10-15 sujeitos em uma sala de aula. Os participantes do estudo receberam o questionário com instruções e recomendações para o seu preenchimento, não sendo estabelecido limite de tempo para o seu preenchimento e as eventuais dúvidas manifestadas pel os adolescentes eram prontamente esclarecidas pelo profissional que acompanhava a coleta de dados. Durante o preenchimento do questionário os adolescentes não se comunicaram entre si, na tentativa de minimizar possíveis interferências indesejáveis em suas respostas.

As informações acerca da reprodutibilidade do questionário foram obtidas mediante uma segunda aplicação nos mesmos sujeitos, com intervalo de duas semanas, utilizando-se de procedimentos idênticos aqueles adotados na primeira aplicação. Aqueles adolescentes que não compareceram em dia e hora agendados para o preenchimento da réplica do questionário não foram considerados no estudo.

Após preencher a réplica do questionário, os partici- 
pantes do estudo receberam instrumento retrospectivo de auto-recordação das atividades diárias ( $\mathrm{R}$ 24h) preconizado por Bouchard et al. (9), com instruções e recomendações no sentido de identificar e registrar o tipo de atividade realizada ao longo do dia. Nesse caso, as atividades do cotidiano são classificadas em um continuum envolvendo nove categorias, de acordo com estimativas quanto ao custo calórico médio das atividades realizadas por humanos: (1) repouso na cama; (2) atividades realizadas em posição sentada; (3) atividades leves realizadas em posição em pé; (4) atividades que exigem caminhadas leves ( $<4 \mathrm{~km} / \mathrm{hora}$ ); (5) trabal ho manual leve; (6) atividades de lazer ativo e prática de esportes recreativos; (7) trabalho manual em ritmo moderado; (8) atividades de lazer ativo e prática de esportes de intensidade moderada; e (9) trabal ho manual intenso e prática de esportes competitivos.

Para o seu preenchimento, o dia foi dividido em 96 períodos de 15 minutos cada, e os participantes do estudo identificaram o tipo de atividade, classificada entre as categorias 1 e 9, realizada em cada período de 15 minutos, durante as 24 horas do dia. Lista de atividades características do cotidiano, exemplificando atividades contempladas nas diferentes categorias, foi apresentada aos adolescentes na tentativa de facilitar o preenchimento do instrumento. Ainda, os participantes do estudo foram instruídos a realizarem anotações adicionais, caso al guma atividade realizada não estava sendo contemplada na lista especificamente elaborada para essa finalidade.

De posse dessas informações, foi determinado o tempo despendido por cada participante do estudo nas diferentes categorias de atividade física e, com base nas referências calóricas sugeridas pelos idealizadores do instrumento de medida utilizado (9), estabeleceram-se estimativas quanto ao dispêndio energético por quilograma de peso corporal das atividades real izadas ao longo de cada dia ( $\mathrm{kcal} / \mathrm{kg} / \mathrm{dia}$ ) como indicador da prática de atividade física cotidiana. $O$ instrumento retrospectivo foi preenchido pelos adolescentes em quatro dias da mesma semana, dois no meio (entre segunda e sexta-feira) e dois no final de semana (sábado e domingo). Para efeito de cálculo, utilizou-se média ponderada envolvendo os dois dias do meio e do final de semana.
O tratamento estatístico foi realizado mediante o pacote computadorizado Statistical Package for the Social Science (SPSS) - versão 13.0. Os dados foram inicialmente comparados com a curva normal utilizando o teste de Kolmogorov-Smirnov (K-S).

Somente os valores equivalentes ao dispêndio energético/dia mostraram distribuição assimétrica (K-S $=0,781 ; p<0,0001$ entre as moças e K-S = 0,632; $p<0,0001$ entre os rapazes). Os valores equivalentes às dimensões da atividade física atribuídos mediante o BQHPA mostraram distribuição de freqüência normal. Para a caracterização da amostra selecionada para o estudo recorreu-se aos procedimentos da estatística descritiva (média \pm desviopadrão). Informações quanto à reprodutibilidade do questionário foram anal isadas mediante dois procedimentos associados ao índice de concordância entre réplicas de medidas: (a) coeficiente de correl ação intraclasse ( $R$ ); e (b) plotagem em diagrama de dispersão de acordo com técnica desenvolvida por Bland \& Altman (6). Com relação à validade do questionário, recorreu-se ao teste de proporção de concordância de Kappa entre categorizações de acordo com distribuições de quartis dos indicadores de atividade física produzidos pelos escores da AFHabitual e pelas estimativas do dispêndio energético médio das atividades realizadas ao longo do dia ( $\mathrm{kcal} / \mathrm{kg} / \mathrm{dia}$ ) relatadas pelos adolescentes mediante o R-24h.

\section{RESULTADOS}

Informações estatísticas quanto às características antropométricas, aos escores equivalentes às dimensões da atividade física, atribuídos mediante o BQHPA, e às estimativas associadas ao dispêndio energético relacionadas às atividades do cotidiano, estabel ecidas mediante o R-24h, são mostradas na tabela 1. 
Tabela 1. Valores médios e desvios-padrão das características antropométricas e dos indicadores relacionados às dimensões da atividade física dos adolescentes envolvidos no estudo.

\begin{tabular}{|c|c|c|c|c|}
\hline & \multicolumn{2}{|c|}{ Moças } & \multicolumn{2}{|c|}{ Rapazes } \\
\hline & $\begin{array}{c}\leq 14 \text { Anos } \\
{[n=59]}\end{array}$ & $\begin{array}{c}>14 \text { Anos } \\
{[n=33]}\end{array}$ & $\begin{array}{c}\leq 14 \text { Anos } \\
{[n=38]}\end{array}$ & $\begin{array}{l}>14 \text { Anos } \\
{[n=31)}\end{array}$ \\
\hline Idade [anos] & $12,92 \pm 0,86$ & $15,88 \pm 0,93$ & $13,00 \pm 0,81$ & $15,81 \pm 0,70$ \\
\hline Estatura $(\mathrm{cm})$ & $158,27 \pm 7,05$ & $161,16 \pm 5,54$ & $162,17 \pm 9,34$ & $175,56 \pm 8,59$ \\
\hline Peso Corporal $(\mathrm{kg})$ & $49,75 \pm 8,34$ & $55,54 \pm 12,16$ & $55,99 \pm 15,14$ & $65,63 \pm 12,73$ \\
\hline $\operatorname{IMC}\left(\mathrm{kg} / \mathrm{m}^{2}\right)$ & $19,83 \pm 2,93$ & $21,30 \pm 3,98$ & $21,11 \pm 4,92$ & $21,42 \pm 3,25$ \\
\hline \multicolumn{5}{|l|}{ BQHPA } \\
\hline AFOcupacional & $2,36 \pm 0,32$ & $2,33 \pm 0,5 ?$ & $2,57 \pm 0,72$ & $2,35 \pm 0,48$ \\
\hline AFEsporte & $2,70 \pm 1,03$ & $2,46 \pm 1,01$ & $3,39 \pm 1,23$ & $2,94 \pm 0,85$ \\
\hline AFLocomoção & $2,64 \pm 0,69$ & $2,52 \pm 0,46$ & $2,98 \pm 0,73$ & $2,95 \pm 0,66$ \\
\hline AFHabitual & $7,70 \pm 1,47$ & $7,32 \pm 1,44$ & $8,94 \pm 1,55$ & $8,24 \pm 1,41$ \\
\hline \multicolumn{5}{|l|}{$\mathrm{R}-24 \mathrm{~h}$} \\
\hline $\begin{array}{l}\text { Dispêndio Energético } \\
\text { (Kcal/kg/dia) }\end{array}$ & $43,63 \pm 5,08$ & $44,03 \pm 5,07$ & $48,90 \pm 6,38$ & $43,20 \pm 5,28$ \\
\hline
\end{tabular}

Os $\mathrm{R}$ associados aos escores equivalentes às diferentes dimensões da atividade física apresentados pelos adolescentes em réplicas de aplicação do BQHPA são disponibilizados na tabela 2 . Os valores encontrados demonstram magnitudes entre 0,55 e 0,82 . Ao estratificar por sexo e por grupo etário, observa-se que os rapazes e os adolescentes com mais idade tendem a apresentar maiores concordâncias entre as réplicas. Análise específica das dimensões da atividade física tratadas no questionário revela que os valores de $\mathrm{R}$ quanto à AFEsporte tendem a ser mais elevados que nas demais dimensões. A capacidade dos adolescentes em apresentar informações quanto à AFOcupacional é a que apresenta menores val ores de $\mathrm{R}$.

Tabela 2. Coeficientes de correlação intra-classe e intervalo de confiança de $95 \%$ associados aos escores das dimensões de atividade física estabelecidos mediante réplicas de aplicação do BQHPA em adolescentes.

\begin{tabular}{lcclcc}
\hline & \multicolumn{2}{c}{ Moças } & & \multicolumn{2}{c}{ Rapazes } \\
\cline { 2 - 3 } \cline { 6 - 7 } & \multicolumn{1}{c}{$\leq 14$ Anos } & $>14$ Anos & & $\leq 14$ Anos & $>14$ Anos \\
\hline AFOcupacional & $0,55(0,48-0,63)$ & $0,61(0,55-0,68)$ & & $0,68(0,60-0,78)$ & $0,69(0,63-0,77)$ \\
AFEsporte & $0,79(0,71-0,89)$ & $0,85(0,81-0,89)$ & & $0,73(0,66-0,82)$ & $0,82(0,78-0,87)$ \\
AFLocomoção & $0,61(0,56-0,67)$ & $0,70(0,64-0,77)$ & & $0,71(0,65-0,77)$ & $0,76(0,71-0,83)$ \\
AFHabitual & $0,66(0,58-0,76)$ & $0,76(0,70-0,83)$ & & $0,75(0,68-0,83)$ & $0,80[0,74-0,87)$ \\
\hline
\end{tabular}

Na figura 1 são ilustrados os diagramas de dispersão com a plotagem dos valores médios do escore relacionado à AFHabitual, calculados mediante informações apresentadas pelos adolescentes em réplicas de aplicação do BQHPA (abscissa), e as diferenças individuais entre ambas as aplicações (ordenada). Este procedimento de análise proposto por Bland \& Altman (6) permite visualizar as diferenças médias e os limites extremos de concordância ( \pm 2 desvios-padrão da diferença) apresentados pelas réplicas de aplicação do questionário. A disposição gráfica dos dados sugere maior variabilidade entre as moças e nos grupos etários mais jovens ( $\leq 14$ anos). Porém, nos quatro subgrupos considerados, os adolescentes apresentam importantes coincidências entre as réplicas de aplica ção do questionário. As diferenças médias e os limites de concordância variam entre $0,0 \pm 1,4$ (rapazes $>14$ anos) e 0,0 $\pm 2,0$ (moças $\leq 14$ anos). 
Ordenada: diferenças individuais entre réplicas de aplicação. Abscissa: valores médios entre a primeira e a segunda aplicação.

$\leq 14$ Anos

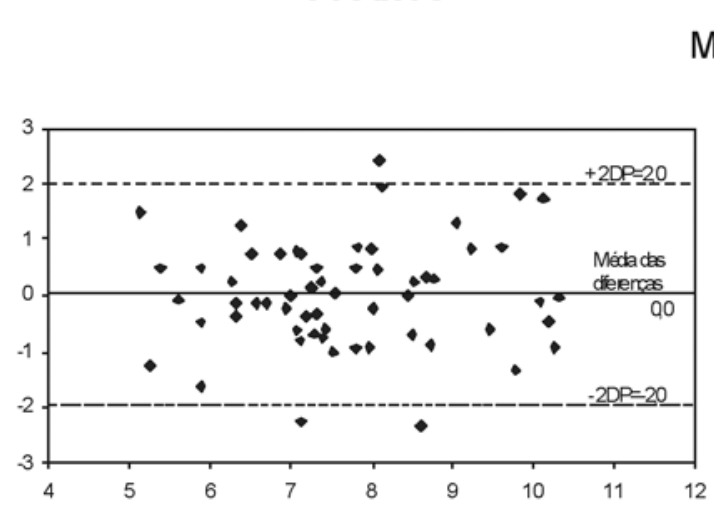

$>14$ Anos

\section{Moças}

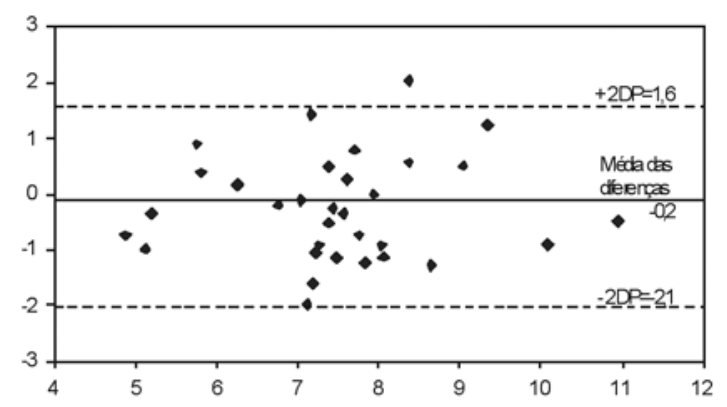

Rapazes
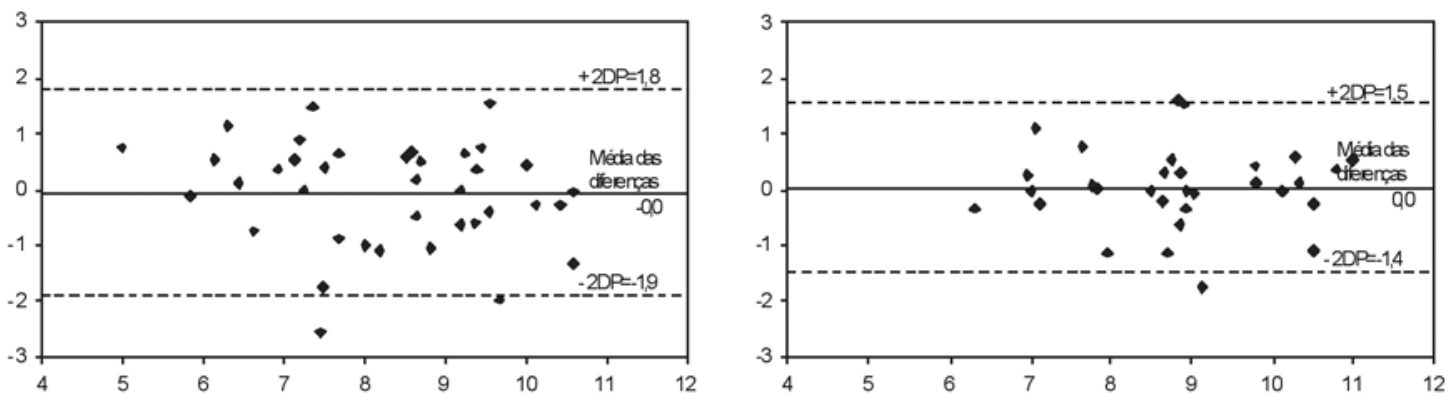

Figura 1. Plotagem de Bland-Altman para os limites de concordância entre réplicas de aplicação do questionário Baecke para avaliação da atividade física habitual em adolescentes.

$\mathrm{Na}$ tabela 3 encontram-se os indicadores estatísticos relacionados à validade das informações provenientes do BQHPA diante do R-24h. Informações ajustadas por sexo e por grupo etário quanto aos índices Kappa entre os escores da AFHabitual e as estimativas do dispêndio energético/ dia revelam valores com significância estatística. Com relação à proporção de concordância, quando os escores da AFHabitual e as estimativas do dispêndio energético/ dia são categorizados em quartis, verifica-se que entre 33,5 e 76,6\% dos adolescentes são reunidos em um mesmo quartil. A proporção de concordâncias mais elevada é encontrada entre os rapazes e no grupo etário com mais idade (> 14 anos).

Tabela 3. Indicadores estatísticos relacionados à validade das informações provenientes do BQHPA diante do R-24h relatadas pelos adolescentes.

\begin{tabular}{|c|c|c|c|c|}
\hline & \multicolumn{2}{|c|}{ Moças } & \multicolumn{2}{|c|}{ Rapazes } \\
\hline & $\leq 14$ Anos & $>14$ Anos & $\leq 14$ Anos & $>14$ Anos \\
\hline Kappa & 0,36 & 0,46 & 0,41 & 0,59 \\
\hline Erro Padrão & 0,07 & 0,05 & 0,06 & 0,02 \\
\hline $\mathrm{p}$ & 0,048 & 0,021 & 0,039 & 0,001 \\
\hline Concordância (\%) & 33,5 & 57,0 & 42,9 & 76,6 \\
\hline
\end{tabular}




\section{DISCUSSÃO}

Em razão da facilidade de aplicação em grandes grupos de sujeitos, do menor custo e por permitir coletar informações com relação ao tipo e ao contexto em que são realizadas, o que não ocorre com os demais recursos de medida, os questionários representam o instrumento mais acessível para a avaliação da atividade física habitual, sobretudo em estudos de natureza epidemiológica. No entanto, por conta das características das informações que se propõem a observar, podem apresentar inconsistências nas respostas e, quando comparados com outros recursos de medida, demonstrar maior dificuldade para atender os critérios associados à reprodutibilidade e à validade de seus resultados.

A reprodutibilidade do questionário em questão no presente estudo foi analisada mediante dois procedimentos estatísticos: coeficiente de correlação intraclasse e plotagens em diagrama de dispersão de acordo com a técnica de Bland \& Altman (6). Com relação aos coeficientes de correlação intraclasse, verifica-se que os valores de $R$ encontrados apresentaram tendências similares às obtidas por outros estudos disponibilizados na literatura envolvendo diferentes tipos de questionários di recionados à avaliação da atividade física em adolescentes (1, 25, 27). Ademais, constata-se que a capacidade de reprodutibilidade encontrada em réplicas de aplicação do BQHPA nos adolescentes selecionados no presente estudo coincide com a encontrada por esse mesmo questionário em sujeitos adultos (22).

Os valores dos coeficientes de correlação intraclasse encontrados no presente estudo são ligeiramente inferiores aos coeficientes de correlação momentoproduto de Pearson observados no estudo original de proposição do BQHPA, envolvendo sujeitos adultos de ambos os sexos entre 20 e 32 anos de idade, com réplicas aplicadas em intervalo de três meses (4). $O$ estudo desenvolvido por Pols et al. (23) relata coeficientes de correlação momento-produto de Pearson entre 0,76 e 0,89, e entre 0,71 e 0,83, para homens e mulheres adultas, respectivamente, com réplicas após cinco meses. Valores de reprodutibilidade mais elevados mediante o coeficiente de correlação intraclasse são encontrados no estudo desenvolvido por Philippaerts \& Lefreve (22), envolvendo homens adultos, com idades entre 30 e 40 anos, com intervalo de um mês entre as aplicações. Nossos resultados apontam menores associações entre os escores provenientes das informações vinculadas à AFOcupacional, diferentemente dos resultados observados nos estudos envolvendo adultos que apontaram menores associações entre os escores representativos das AFLocomoção.

Por outro lado, ao analisar informações adicionais quanto à reprodutibilidade do BQHPA, por intermédio da técnica estatística proposta por Bland \& Altman (6), constatou-se que a capacidade de concordância entre réplicas de aplicação do questionário pode ficar prejudicada. Neste sentido, ao que parece, por conta dos pressupostos estatísticos envolvidos em seus procedimentos de cálculo, a técnica de Bland \& Altman deverá apresentar maior sensibilidade na detecção de diferenças entre réplicas de medidas do que análises convencionais envolvendo o coeficiente de correlação de Spearman. Em tese, assumese que, diferenças sistemáticas eventual mente detectadas entre réplicas de medidas não deverão necessariamente afetar a magnitude dos coeficientes de correlação de maneira significativa; porém, poderão restringir substancialmente a grau de concordância entre ambas as medidas (3), o que pode ser comprovado mediante análise realizada por intermédio da técnica sugerida por Bland \& Altman.

No presente estudo, para anál ise da reprodutibilidade mediante a técnica de plotagem sugerida por Bland \& Altman foram construídos diagramas de dispersão levando-se em consideração a diferença média entre as réplicas do BQHPA e a distribuição das diferenças individuais em um intervalo de confiança de dois desvios-padrão. Em assim sendo, a expectativa é que, ao aplicar o questionário em dois momentos, aos mesmos adolescentes e em situações semelhantes, as diferenças médias entre as réplicas de aplicação devem se aproximar do valor zero e os limites extremos dos interval os de confiança se apresentarem o mais próximo possível dos valores equivalentes às diferenças médias.

No entanto, ao observar as informações disponibilizadas na figura 1 , se por um lado, constata-se que os escores equivalentes às dimensões da AFHabitual consignados mediante o BQHPA apresentaram valores médios entre as réplicas de aplicação do questionário próximos de zero, por outro, verificam-se dis- 
tâncias entre os limites extremos dos intervalos de confiança que podem gerar preocupação.

Para ilustrar a situação, nos quatro subgrupos considerados da amostra selecionada para estudo, as diferenças médias entre os escores observados equivalentes à AFHabitual nas duas aplicações do questionário foram iguais ou muito próximas de zero. Porém, os limites extremos dos intervalos de confiança ( \pm 2 desvios-padrão) estabeleceram-se por volta de duas unidades, o que revela uma variabilidade individual quanto à concordância entre as réplicas de aplicação do questionário demasiadamente elevada, se confrontadas com os valores médios encontrados, entre 7,32 e 8,94 unidades. Em linguagem estatística essas informações apontam que, em 95\% dos casos, um mesmo adolescente, mediante réplicas de aplicação do BQHPA em situações idênticas, pode apresentar escores vinculados à AFHabitual com diferenças de até duas unidades. Mesmo diante dessas evidências, sugere-se cautela na interpretação da maior amplitude observada entre os limites extremos dos intervalos de confiança, considerando o número relativamente reduzido de sujeitos envolvidos no presente estudo e a ocorrência de al guns casos outliers na disposição dos val ores no diagrama de dispersão.

Com relação aos indicadores de validação, a opção por um método de referência que possa servir de comparação torna-se de fundamental importância e ponto crítico para a análise das informações, considerando que este deverá representar com maior acurácia possível a verdadeira medida quanto ao nível de prática da atividade física. Neste sentido, indubitavelmente, os recursos que envolvem a água duplamente marcada são reconhecidos como os mais acurados para a estimativa do dispêndio energético/ dia e, desse modo, define-se como o método de referência mais indicado para oferecer informações quanto à validação de questionários direcionados à aval iação do nível habitual de atividade física (13). Entretanto, as dificuldades operacionais de seus procedimentos e o elevado custo dos exames laboratoriais que esse método exige torna-se sua utilização proibitiva em pesquisas com maior número de sujeitos. Em vista disso, optou-se por empregar como método de referência no presente estudo o R-24h de atividades do cotidiano preconizado por Bouchard et al. (9), devido ao fato de depender minimamente da memória do avaliado e prover informações detal hadas quanto às atividades realizadas ao longo de cada dia, classificadas em nove categorias de acordo com o tipo e a intensidade dos esforços físicos exigidos. Os indicadores estatísticos relacionados à validade encontrados entre os adolescentes com mais de 14 anos de idade aqui analisados se aproximam das tendências observadas em estudos disponibilizados na literatura envolvendo a aplicação do BQHPA em sujeitos adultos (22, 23), e são consistentes com informações provenientes de outras propostas de questionários di recionados à avaliação da atividade física habitual em adolescentes $(1,25,27)$. Porém, ao considerar as informações associadas aos adolescentes com menos de 14 anos de idade, verifica-se que, apesar de apresentarem significância estatística, os valores equivalentes ao índice Kappa encontrados no presente estudo são de magnitude inferiores àqueles apresentados por outros estudos envolvendo diferentes propostas de questionários e informações provenientes de $\mathrm{R}-24 \mathrm{~h}(7,33)$.

Neste particular, o formato do BQHPA solicita do aval iado que procure dimensionar atributos vinculados à prática habitual de atividade física mediante uma escal a contínua do tipo Lickert. Em sendo assim, ao assumir que a capacidade de percepção quanto à intensidade, à duração e à freqüência com que são realizadas determinadas atividades do cotidiano torna-se mais sensível e aprimorada com o passar dos anos na infância e na adolescência (24), é de se esperar que os adolescentes com mais idade venham a apresentar maior associação e concordância entre as informações apresentadas pelo BQHPA e pelo R-24h. Essas evidências reforçam hipótese sugerida por Trost et al. (29) no sentido de que, além de atributos vinculados aos aspectos socioculturais e educacionais, informações quanto à idade e ao gênero são algumas das preocupações que devem ser consideradas na escolha e na aplicação de questionários direcionados à aval iação da prática habitual de atividade física.

\section{CONCLUSÕES}

O BQHPA tem a vantagem de ser curto, de fácil aplicação e de apresentar aos avaliados questões com opções de respostas amigáveis, e, portanto, em tese, 
de ser mais exeqüível para utilização na população jovem. Porém, demanda interpretações subjetivas quanto às definições da intensidade, da duração e da freqüência das atividades físicas habitualmente praticadas, o que pode, eventualmente, dependendo do contexto em que os avaliados estão inseridos, comprometer a qualidade das informações oferecidas para análise. Em assim sendo, disponibilizar indicadores quanto à reprodutibilidade e à validade deste questionário em amostras de sujeitos pertencentes a segmentos específicos da população, torna-se de fundamental importância para o desenvolvimento de futuros estudos envolvendo a prática habitual de atividade física.

Diante dos resultados encontrados no presente estudo pode-se inferir que, mediante procedimentos estatísticos mais conservadores, as informações apresentadas pel os adolescentes mediante a utilização do BQHPA apresentaram satisfatória capacidade de concordância entre réplicas de sua aplicação. No entanto, ao considerar recurso estatístico aparentemente de maior sensibilidade na detecção de diferenças entre réplicas de aplicação do questionário, verifica-se a ocorrência de variações que podem fragilizar sua capacidade de reprodutibilidade, e, por conseqüência, sua potencialidade para acompanhar com maior confiabilidade a prática habitual de atividade física em adolescentes. Quanto aos indicadores relacionados à validade, embora o instrumento critério utilizado para análise, o R-24h, possa não ser considerado padrão-ouro, as informações estatísticas selecionadas no estudo indicam que o BQHPA pode se definir como um instrumento válido direcionado ao acompanhamento da prática habitual de atividade física, sobretudo entre os adolescentes com mais idade (> 14 anos).

Chama-se atenção para o fato das informações aqui reunidas, ao serem generalizadas para outras populações de jovem, sejam realizadas com as devidas precauções. O presente estudo foi conduzido envolvendo uma amostra de adolescentes que apresenta um background sociocultural e educacional específico da população a que pertence. Portanto, não se pode descartar a possibilidade do BQHPA apresentar diferentes comportamentos quanto aos indicadores de reprodutibilidade e de validade ao ser aplicado em adolescentes pertencentes a outras populações.

\section{CORRESPONDÊNCIA}

\section{Dartagnan Pinto Guedes}

Rua Ildefonso Werner 177

Condomínio Royal Golf

CEP 86001-970 - Londrina - Paraná

darta@ sercomtel.com.br 
REFERÊNCIAS BIBLIOGRÁFICAS

1. Aaron DJ, Kriska AM, Dearwater SR, Cauley JA, Metz KF, LaPorte RE (1995). Reproducibility and validity of an epidemiologic questionnaire to assess past year physical activity in adolescents. A m J Epidemiol 142:191-201

2. Albanes D, Conway JM, Taylor PR, Moe PW, Judd J (1990). Validation and comparison of eight physical activity questionnaires. Epidemiology 1:65-71

3. Atkinson G, Nevill AM (1998). Statistical methods for assessing measurement error (reliability) in variables relevant to sports medicine. Sports M ed 26:217-38

4. Baecke JAH, Burema J, Frijters JER (1982). A short questionnaire for the measurement of habitual physical activity in epidemiological studies. A m J Clin N utr 36:936-42

5. Blair S, Connelly JC (1996). How much physical activity should we do? The case for moderate amounts and intensities of physical activity. Res Q Exerc Sport 67:193-205

6. Bland JM, Altman DG (1986). Statistical methods for assessing agreement between two methods for clinical measurement. Lancet 8:307-10

7. Booth ML, Okely AD, Chey TM, Bauman A (2002). The reliability and validity of the Adolescent Physical Activity Recall Questionnaire. M ed Sci Sports Exerc 34:1986-95

8. Bouchard C, Shephard RJ, Stephns T (1994). Physical Activity, Fitness and Health: International Proceedings and Consensus Statement. Champaign, Illinois; Human Kinetics.

9. Bouchard C, Tremblay A, LeBlanc C, Lortie G, Sauard R, Therialt $G$ (1983). A method to assess energy expenditure in children and adults. A m J Clin N utr 37:461-7

10. Calfas KJ, Taylor WC (1994). Effects of physical activity on psychological variables in adolescents. Pediat Exerc Sci 6:406-23

11. Caspersen CJ, Powell KE, Christenson GM (1985). Physical activity, exercise, and physical fitness: definitions and distinctions for health-related research. Public $\mathrm{H}$ ealth Rep 100:126-31

12. Eisenmann JC (2004). Physical activity and cardiovascular disease risk factors in children and adolescents: an overview. Can I Cardiol 20:295-301

13. Kohl HW, Fulton JE, Caspersen CJ (2000). Assessment of physical activity among children and adolescents: a review and synthesis. Prev M ed 31:S54-S76

14. Kriska AM, Caspersen CJ (1997). Introduction to a collection of physical activity questionnaires. M ed Sci Sports Exerc 29:S5-S9

15. Laakso L, Viikari J (1997). Physical activity in childhood and adolescence as predictor of physical activity in young adulthood. A m J Prev M ed 13:317-23

16. Maia JAR, Loos R, Beunen G, Thomis $M$, Vlietinck $R$, Morais FP, Lopes VP (1999). Aspectos genéticos da prática desportiva: um estudo em gêmeos. Rev Paul Educ Fis 13:160-76

17. Maia JAR, Thomis M, Beunen G (2002). Genetic factors in physical activity levels: a twin study. Am J Prev Med 23(2 Suppl) :87-91
18. Miller DL, Freedson PS, Kline GM (1994). Comparison of activity levels using Caltrac accelerometer and five questionnaires. Med Sci Sports Exerc 26:376-82

19. Oliveira MMC, Maia JAR, Lopes VP, Seabre A, Garganta R (2003). Aspectos genéticos da actividade física: um estudo multimodal em gêmeos monozigóticos e dizigóticos. Rev Paul Educ Fis 17:104-18

20. Park J (2003). Adolescent self-concept and health into adulthood. Health Rep 14:41-52

21. Pate RR, Pratt M, Blair S, Haskell W, Macera C, Bouchard C, Ettinger W, Helth G, King A (1995). Physical activity and the public health: A recommendation from the Centers for Disease Control and Prevention and the American College of Sports Medicine. JA M A 273:402-7

22. Philippaerts RM, LefevreJ (1998). Reliability and validity of three physical activity questionnaires in Flemish males. A m J Epidemiol 147:982-90

23. Pols MA, Peeters PHM, Bueno-de-Mesquita HB, Ocké MC, Wentink CA, Kemper HCG, Collette HJA (1995). Validity and repeatability of a modified Baecke Questionnaire on Physical Activity. Int J Epidemiol 24:381-7

24. Sallis JF (1991). Self-report measures of children's physical activity. J Sch Health 61:215-9

25. Sallis JF, Buono MJ, Roby J (1993). Seven-day recall and other physical activity self-report in children and adolescents. M ed Sci Sports Exerc 25:99-108

26. Sallis JF, Patrick K (1994). Physical activity guidelines for adolescents: consensus statement. Pediat Exerc Sci 6:302-14

27. Sallis JF, Saelens BE (2000). Assessment of physical activity by self-report: status, limitations, and future directions. Res Q Exerc Sport 71:1-14

28. Taylor WC, Blair SN, Cummings SS, Wun CC, Malina RM (1999). Childhood and adolescent physical activity patterns and adult physical activity. M ed Sci Sports Exerc 31:118-23

29. Trost SG, Pate RR, Sallis JF, Freedson PS, Taylor WC, Dowda M, Sirard J (2002). Age and gender differences in objectively measured physical activity in youth. M ed Sci Sports Exerc 34:350-5

30. Twisk JW, Kemper HC, van Mechelen W (2000). Tracking of activity and fitness and the relationship with cardiovascular disease risk factors. M ed Sci Sports Exerc 32:1456-61

31. US Department of Health and Human Services (1996). Physical Activity and Health: A Report of the Surgeon General. Atlanta, GA; US Department of Health and Human Services. Centers for Disease Control and Prevention, National Center for Chronic Disease Prevention and Health Promotion.

32. Welk GJ, Corbin CB, Dale D (2000). Measurement issues in the assessment of physical activity in children. Res $Q$ Exerc Sport 71:59-73

33. Weston A, Petoso R, Pate RR (1997). Validation of an instrument for measurement of physical activity in youth. Med Sci Sports Exerc 29:138-43 